\title{
Excitonic optical transitions characterized by Raman excitation profiles in single-walled carbon nanotubes
}

\author{
H. N. Tran, ${ }^{1}$ J.-C. Blancon, ${ }^{2}$ J.-R. Huntzinger, ${ }^{1}$ R. Arenal,${ }^{3,4}$ V. N. Popov, ${ }^{5}$ A. A. Zahab, ${ }^{1}$ A. Ayari, ${ }^{2}$ A. San-Miguel, ${ }^{2}$ \\ F. Vallée, ${ }^{2}$ N. Del Fatti, ${ }^{2}$ J.-L. Sauvajol, ${ }^{1}$ and M. Paillet ${ }^{1}$ \\ ${ }^{1}$ Laboratoire Charles Coulomb (UMR5221), CNRS-Université de Montpellier, F-34095 Montpellier, France \\ ${ }^{2}$ Institut Lumière Matière (UMR5306), CNRS-Université Lyon 1, Université de Lyon, F-69622 Villeurbanne, France \\ ${ }^{3}$ Laboratorio de Microscopí-as Avanzadas, Instituto de Nanociencia de Aragón, Universidad de Zaragoza, \\ 50018 Zaragoza, Spain \\ ${ }^{4}$ ARAID Foundation, 50018 Zaragoza, Spain \\ ${ }^{5}$ Faculty of Physics, University of Sofia, BG-1164 Sofia, Bulgaria
}

(Received 19 May 2016; published 22 August 2016)

\begin{abstract}
We examine the excitonic nature of the $E_{33}$ optical transition of the individual free-standing index-identified $(23,7)$ single-walled carbon nanotube by means of the measurements of its radial-breathing-mode and $G$-mode Raman excitation profiles. We confirm that it is impossible to determine unambiguously the nature of its $E_{33}$ optical transition (excitonic vs band to band) based only on the excitation profiles. Nevertheless, by combining Raman scattering, Rayleigh scattering, and optical absorption measurements on strictly the same individual $(23,7)$ single-walled carbon nanotube, we show that the absorption, Rayleigh spectra, and Raman excitation profiles of the longitudinal and transverse $G$ modes are best fitted by considering the nature of the $E_{33}$ transition as excitonic. The fit of the three sets of data gives close values of the transition energy $E_{33}$ and damping parameter $\Gamma_{33}$. This comparison shows that the fit of the Raman excitation profiles provides with good accuracy the energy and damping parameter of the excitonic optical transitions in single-walled carbon nanotubes.
\end{abstract}

DOI: 10.1103/PhysRevB.94.075430

\section{INTRODUCTION}

Single-walled carbon nanotubes (SWNTs) represent one of the best-known one-dimensional (1D) materials for the study of exciton photophysics. For a long time, excitonic effects have been identified as playing a central role in defining the optical properties of SWNTs (for a review, see Ref. [1] and references therein). It has been argued theoretically that the optical transition energy of a SWNT can be written as the sum of a one-particle energy term and a many-body one, which in turn is decomposed into selfenergy, due to electron-electron interactions, and an exciton binding energy [2-6]. These predictions have been validated by means of various experiments, including two-photon absorption [7,8], photoluminescence excitation experiments on semiconducting SWNTs (S-SWNT) [9], absorption experiments on S-SWNTs and on metallic SWNTs (M-SWNT) [10-12], Rayleigh scattering experiments [13-15], scanning tunneling spectroscopy [16], and, recently, ultrafast terahertz spectroscopy [17].

Raman spectroscopy experiments, performed on isolated individual free-standing index-identified $(n, m)$ SWNTs, give valuable information about the intrinsic features of the totally symmetric modes: the radial breathing modes (RBMs) and tangential modes (so-called $G$ modes) [18-21]. Additionally, the measurements of the evolution of the Raman intensity as a function of the excitation energy, the so-called Raman excitation profiles (REPs), of the RBM and the $G$ modes, with laser light polarization along the nanotube axis, allow for the determination of the main characteristics of the optical transitions, namely, the transition energies $E_{i i}$ and the corresponding damping parameters $\Gamma_{i i}(i i=11,22,33, \ldots)$ arising from electronic excitation relaxation processes. As a reminder, the $E_{i i}$ transition energies are named after the corresponding one-particle terms associated with the dipolarallowed transitions between van Hove electron density of states peaks. In principle, the comparison of the experimental REPs of these modes with the theoretical ones could give insight into the nature of the optical transitions, band to band or excitonic, because the theoretical REPs have different shapes in both cases (see Ref. [22] and references therein). Nevertheless, it is usually possible to achieve reasonable fits of the experimental REPs with both theoretical expressions with $E_{i i}$ and $\Gamma_{i i}$ as free parameters [22]. Therefore, it is impossible to determine unambiguously the nature of the optical transitions based only on the analysis of the experimental REPs.

In this paper, we combine optical absorption, Rayleigh scattering, and Raman scattering studies on the same individual free-standing index-identified $(23,7)$ SWNT with a diameter of $2.13 \mathrm{~nm}$. Especially, we discuss the REPs of the RBMs and the $G$ modes measured on this individual SWNT. This has been achieved by employing more than 30 laser excitation lines in the range $E_{L}=1.55-2.0 \mathrm{eV}$, i.e., around the $(23,7)$ $E_{33}$ transition. By considering the nature of the transition as excitonic, we are able to fit, in a consistent way, the absorption and Rayleigh spectra as well as the Raman excitation profiles of the longitudinal (LO) and transverse (TO) $G$ modes of the $(23,7)$ SWNT. The fit of the three sets of independent data gives close values of the transition energy $E_{33}$ and damping parameter $\Gamma_{33}$. This comparison demonstrates that, with the excitonic nature of the transition being determined independently by absorption or Rayleigh spectroscopy, the fit of the Raman excitation profile is able to provide, with good accuracy, the energy and damping parameter of the optical transition.

The experimental information is described in Sec. II. The results are presented and discussed in Sec. III. 


\section{SAMPLE AND EXPERIMENTAL METHODS}

Ultralong individual single-walled nanotubes were grown using the catalytic chemical vapor deposition (CCVD) method directly on homemade slits fabricated by wet etching of a $\mathrm{Si}_{x} \mathrm{~N}_{y} / \mathrm{Si}$ wafer (for details, see Ref. [23]). High-resolution transmission electronic microscopy (HRTEM) images and electron diffraction (ED) patterns were recorded by a FEI Titan microscope operating at $80 \mathrm{kV}$ and within short acquisition times (less than $5 \mathrm{~s}$ for ED) to reduce damage induced by electron diffraction [24]. Direct measurements of the absolute absorption cross-section spectra of freely suspended individual SWNTs were assessed via a spatial modulation spectroscopy technique (for details, see Refs. [12,25]). The resonant Raman scattering measurements were performed with a homemade setup, including an iHR-550 Horiba spectrometer equipped with a liquid-nitrogen-cooled silicon CCD detector, with a wide variety of laser excitation wavelengths using $\mathrm{Ar}^{+}, \mathrm{Kr}^{+}$, and $\mathrm{He}-\mathrm{Ne}$ lasers and tunable Ti:sapphire and dye lasers filtered using tunable laser line filters [26]. The scattered light was collected through a $100 \times$ objective (numerical aperture of 0.95 ) using a backscattering configuration. The laser power impinging the sample was kept below $100 \mu \mathrm{W}$. In these conditions, with a laser spot FWHM of about $500 \mathrm{~nm}$, heating effects are avoided. In all the measurements, both incident and scattered light polarizations are along the nanotube axis. The REPs of the RBMs and the $G$ modes have been measured in the 1.55 to $2.0 \mathrm{eV}$ excitation range. To obtain the REP, the experimental intensity of each mode measured at a specific excitation energy was corrected by the total transmission of our optical system and normalized by the intensity of the $521 \mathrm{~cm}^{-1}$ line of silicon which was taken as a reference sample. We further checked that, in the studied laser energy range, the silicon sample intensity exhibits a dependency similar to $\mathrm{CaF}_{2}$, diamond, or graphite. Rayleigh spectra of individual free-standing SWNTs were measured in a backscattering geometry using a cross-polarization scheme [27] with a Fianium supercontinuum laser as a light source and a fiber-fitted QE-Pro Ocean Optics spectrometer for detection.

\section{RESULTS AND DISCUSSION}

An individual free-standing SWNT, suspended across a trench [see the image of this tube in Fig. 1(a), inset], has been unambiguously index identified as $(23,7)$ by electronic diffraction [Fig. 1(a), left half of the figure]. The simulated electron diffraction pattern of this SWNT is shown in the right half of Fig. 1(a).

The absorption spectrum of the $(23,7)$ SWNT, measured in the 1.4 to $2.4 \mathrm{eV}$ range, is displayed in Fig. 1(b). Two transitions are identified: $E_{33}$ at $1.66 \mathrm{eV}$ and $E_{44}$ at $2.21 \mathrm{eV}$ [12].

Raman experiments have been performed in the excitation range $E_{L}=1.55-2.0 \mathrm{eV}$. In this range, only the $E_{33}$ transition is present [see Fig. 1(b)].

The $E_{44}$ transition, located at $2.21 \mathrm{eV}$, is far from the outgoing resonance of the $E_{33}$ transition, i.e., far from $E_{33}+E_{G}$ with $E_{33}=1.66 \mathrm{eV}$ and $E_{G} \approx 0.2 \mathrm{eV}$, where $E_{G}$ is the average energy of the $G$ modes. Consequently, no quantum interference effect between different transitions can contribute to the Raman intensity [22,28,29]. In this case, and as expected, the RBM does not appear in the Raman spectrum excited at $E_{L}=1.85 \mathrm{eV}$, which is far from the $E_{33}$ transition. Conversely, a strong RBM is observed in the Raman spectrum excited at $E_{L}=1.66 \mathrm{eV}$ [Fig. 1(c)]. The $G$-mode regions of the Raman spectra excited at $1.66 \mathrm{eV}$ $(1.85 \mathrm{eV})$ correspond to incoming (outgoing) resonance, i.e., $E_{L} \approx E_{33}\left[E_{L} \approx E_{33}+E_{G}\right.$; Fig. 1(c)]. The narrow and symmetric line shape of the LO mode $\left(1591 \mathrm{~cm}^{-1}\right)$ and TO mode $\left(1576.5 \mathrm{~cm}^{-1}\right)$ agrees with the chiral semiconducting nature of the $(23,7)$ SWNT [18].

The experimental REPs of the RBM, LO, and TO $G$ modes are displayed in Fig. 2 (circles). Up to now, REPs of RBM of individual SWNTs have been obtained from non-index-identified SWNTs deposited on a substrate [30] and from samples containing several SWNT species [31,32], and the REPs of $G$ modes were measured only recently after it became possible to produce samples containing enriched species of specific SWNTs [29,33,34]. These results emphasize the importance of the availability of pure-chirality samples, such as isolated individual index-identified SWNTs, for enabling the study of previously inaccessible behaviors in carbon nanotubes. The incoming and outgoing resonances are not resolved in the REP of the RBM (Fig. 2, top). By contrast, for LO and TO $G$ modes the two incoming and outgoing resonances are well separated (Fig. 2, middle and bottom). In addition, it must be emphasized that the intensity of the incoming and outgoing resonances of the REPs of the $G$ modes of this specific $(23,7)$ SWNT are close to each other. However, a significant asymmetry of the incoming and outgoing resonances has been found for other chiralities [33].

Usually, the intensity of the Raman active modes of a SWNT is calculated within a third-order quantum perturbation theory. Depending on the nature of the optical transition, simplified expressions were established to describe the dependence of the Raman intensity on the laser excitation (see Ref. [22] and references therein).

For a band-to-band transition, assuming a parabolic shape of the valence and conduction bands, the following simplified expression of the Raman intensity has been derived [22,35]:

$$
\begin{aligned}
I_{\mathrm{REP}}^{(\mathrm{bb})}\left(E_{L}\right) \propto & \mid \sum_{i} \frac{M_{i i}}{\sqrt{E_{L}-E_{i i}+j \Gamma_{i i}}} \\
& -\left.\frac{M_{i i}}{\sqrt{E_{L}-E_{i i}-E_{p h}+j \Gamma_{i i}}}\right|^{2}
\end{aligned}
$$

where $E_{p h}$ is the energy of the Raman active mode and $j$ is the imaginary unit. Previously, this expression has been used to derive the electron-phonon coupling matrix elements of the RBM [36] and the $G$ modes [37] in SWNTs from their REPs.

For an excitonic transition, a simplified expression of the Raman intensity takes the following form [22]:

$$
\begin{aligned}
& I_{\mathrm{REP}}^{(\mathrm{ex})}\left(E_{L}\right) \\
& \quad \propto\left|\sum_{i} \frac{M_{i i}}{E_{L}-E_{i i}+j \Gamma_{i i}}-\frac{M_{i i}}{E_{L}-E_{i i}-E_{p h}+j \Gamma_{i i}}\right|^{2} .
\end{aligned}
$$


(a)

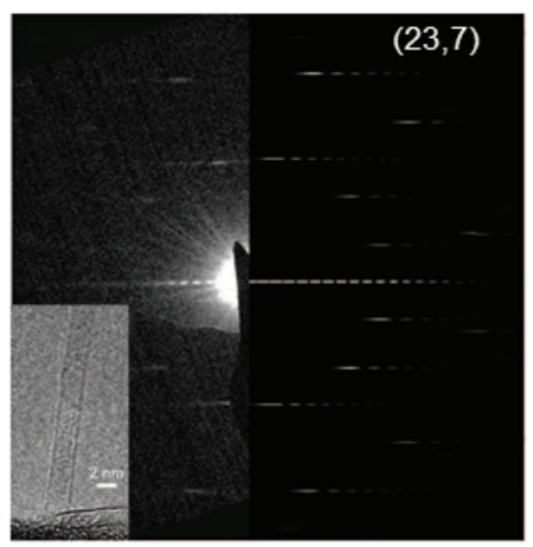

(c)

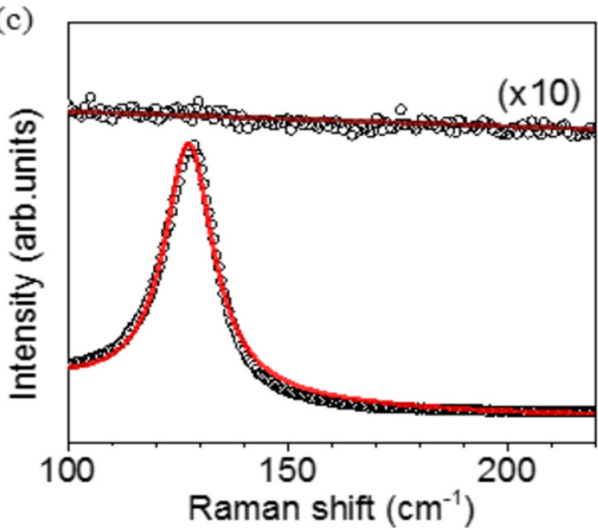

(b)
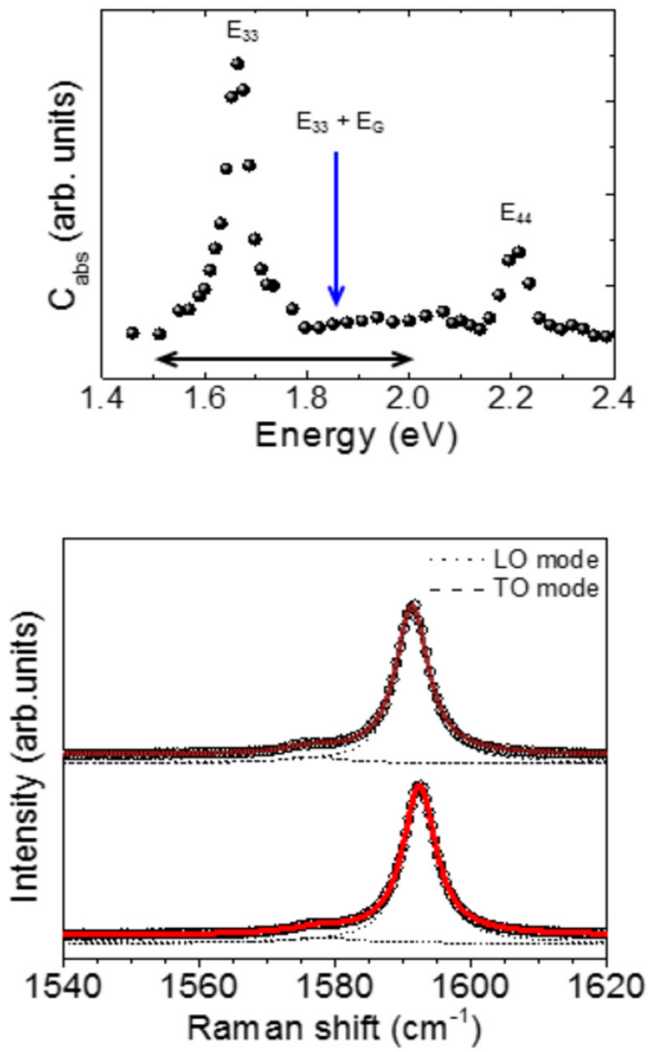

FIG. 1. (a) HRTEM (inset) and electron diffraction (ED) pattern of the $(23,7)$ SWNT, experimental ED pattern (left) and simulated ED pattern (right). (b) Absorption spectrum of the $(23,7)$ SWNT in the 1.4-2.4 eV range; the horizontal double arrow indicates the excitation energy range used in the Raman experiments. (c) RBM and $G$-mode ranges of the Raman spectra of the (23,7) SWNT excited at 1.85 eV (top) and $1.66 \mathrm{eV}$ (bottom). Each spectrum is normalized on the intensity of the LO $G$ mode. The vertical offset of the spectra is for clarity. Dots are experimental data; brown and red solid lines show the fit of the spectra recorded at 1.85 and $1.66 \mathrm{eV}$, respectively; black dotted (dashed) line is the result of the fit of the $\mathrm{LO}$ (TO) $G$ mode by a Lorentzian.

In the denominators of the preceding expressions, the first (second) term describes the incoming (outgoing) resonance. For simplification, $M_{i i}$ condenses the electron-phonon and electron-photon matrix elements involved in the resonance with the $E_{i i}$ transition. It should be noted that both expressions predict the same intensity for the incoming and outgoing resonances. For the $(23,7)$ SWNT, both expressions are good candidates for modeling the REP since the experimental incoming- and outgoing-resonance intensities of the $G$ modes are close indeed. Thus, expressions (1) and (2) are used in the following for the analysis of the experimental REPs.

The REPs of the RBM, LO, and TO $G$ modes are fitted simultaneously by considering $M_{33}, E_{33}$, and $\Gamma_{33}$ as free parameters, with the constraint that $E_{33}$ and $\Gamma_{33}$ have the same values for the three modes. In Fig. 2, the blue solid line (red dotted line) is the fit of the experimental REPs when considering the $E_{33}$ transition to be excitonic (band to band). Because expression (1) gives a simplified description of the band-to-band transition, in Fig. 2 (green dashed line), we also provide a full calculation of the Raman intensity for this case performed within third-order quantum perturbation theory (for details, see Ref. [38]). We note that, in the latter calculation, $\Gamma_{33}$ is fixed at the value of $16 \mathrm{meV}$ determined from the fit in the simplified band-to-band model, and the calculated
REPs are scaled and rigidly shifted in energy to match the experimental transitions. The calculated line shape of the REP is close to that obtained using the simplified expression (1) for the RBM and the LO mode. In the case of the LO mode, the full calculation predicts close intensity for the incoming and outgoing resonances. This agreement justifies, a posteriori, the use of a simplified expression for the fit of the REPs. However, the full calculation of the TO mode predicts a weak asymmetry of the incoming and outgoing resonances which is not seen in the experimental REPs.

We observe that, although the quality of the fit of the REPs is slightly better in the excitonic picture (the coefficient of determination $R^{2}$ is 0.95$)$ than for the band-to-band transition model $\left(R^{2}=0.89\right.$; Fig. 2 , blue solid line and red dotted line), this is not enough to determine unambiguously the nature of the transition from solely the knowledge of the REPs. A detailed analysis of the results of the fits provides the following conclusions: (i) independent of the nature of the transition, the fitted value of $E_{33}$ is $1.664 \pm 0.005 \mathrm{eV}$. (ii) By contrast, the fitted $\Gamma_{33}$ are strongly dependent on the nature of the transition: $\Gamma_{33}=27 \pm 1 \mathrm{meV}$ in the excitonic model, and $\Gamma_{33}=16 \pm$ $1 \mathrm{meV}$ in the band-to-band model (Table I). The lower $\Gamma_{33}$ in the band-to-band model than in the excitonic approach is due to the broader tails of the band-to-band profile. 


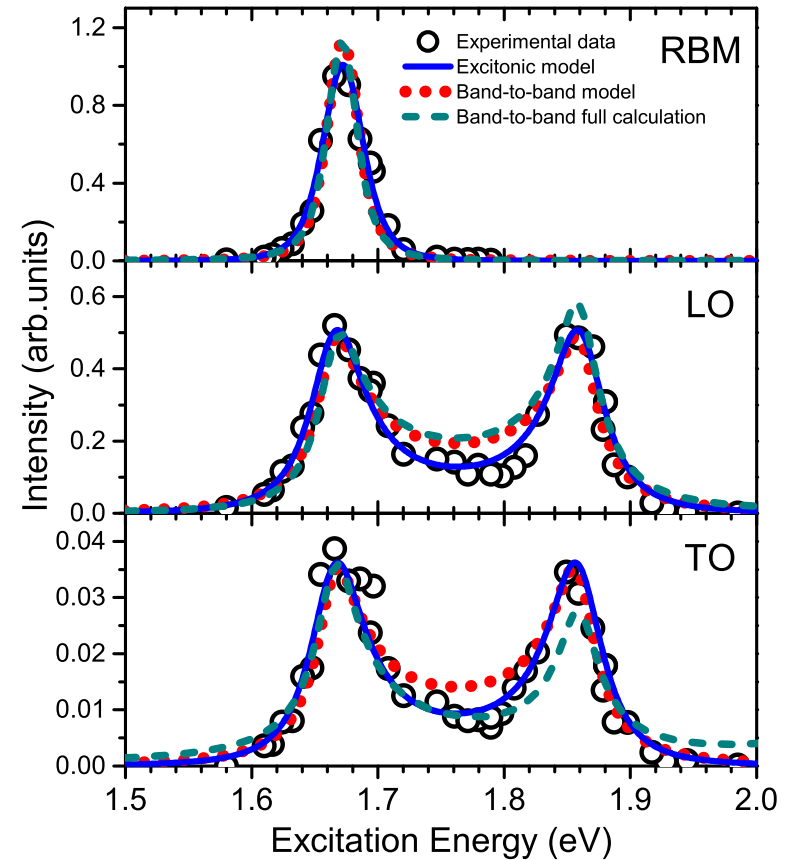

FIG. 2. REPs of the RBM (top), LO $G$ mode (middle), and TO $G$ mode (bottom). Circles are experimental data, with the red dotted line being the best fit for the band-to-band model [expression (1)] and the blue solid line being the best fit for the excitonic model [expression (2)]. $M_{33}, E_{33}$, and $\Gamma_{33}$ are free parameters in the fit (see text). The three REPs were simultaneously fitted in each model. The full calculation of the REP within the band-to-band model is given for comparison (green dashed line).

Rayleigh scattering spectroscopy is known as a powerful technique for studying the optical transitions in SWNTs [13-15]. The Rayleigh scattering spectrum measured around the $E_{33}$ transition of the $(23,7)$ SWNT is displayed in Fig. 3(a) (circles). The fitting of the spectrum is performed following closely the procedure of Ref. [14]. The Rayleigh scattering spectrum is fitted with $E^{3}|\chi(E)|^{2}$, where $\chi(E)$ is the nanotube dielectric susceptibility.

In the excitonic model, close to the $E_{33}$ transition, the susceptibility is approximated by

$$
\chi(E) \propto \frac{1}{E_{33}-E-j \Gamma_{33}} .
$$

TABLE I. Values of $E_{33}$ and $\Gamma_{33}$ derived from the fit of the Raman resonant profiles and Rayleigh and absorption spectra. The coefficient of determination of each fit $R^{2}$ is given.

\begin{tabular}{lccc}
\hline \hline & Raman & Rayleigh & Absorption \\
\hline Excitonic model & & & \\
$E_{33}(\mathrm{eV})$ & $1.664 \pm 0.005$ & $1.659 \pm 0.005$ & $1.664 \pm 0.005$ \\
$\Gamma_{33}(\mathrm{meV})$ & $27 \pm 1$ & $30 \pm 2$ & $26 \pm 2$ \\
$R^{2}$ & 0.95 & 0.97 & 0.99 \\
Band-to-band model & & & \\
$E_{33}(\mathrm{eV})$ & $1.664 \pm 0.005$ & $1.656 \pm 0.005$ & $1.644 \pm 0.010$ \\
$\Gamma_{33}(\mathrm{meV})$ & $16 \pm 1$ & $15 \pm 2$ & $13 \pm 3$ \\
$R^{2}$ & 0.89 & 0.94 & 0.77 \\
\hline \hline
\end{tabular}
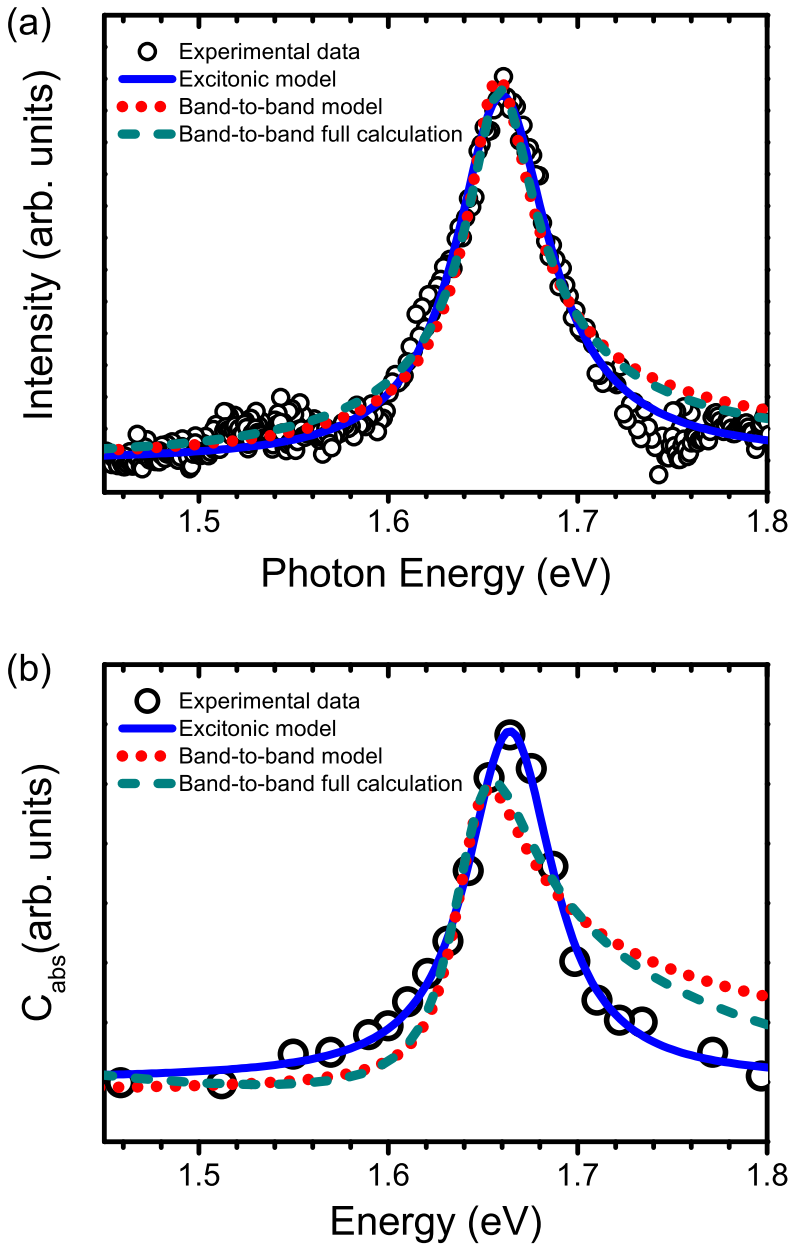

FIG. 3. (a) Rayleigh spectrum in the $1.45-1.8 \mathrm{eV}$ range. Circles are the experimental data. The blue solid line is the fit of the $E_{33}$ peak in the excitonic model; the red dotted line is the best fit of the experimental data in the simplified band-to-band approach. The Rayleigh spectrum obtained by full calculation in the band-to-band model, using a fixed damping parameter of $16 \mathrm{meV}$ and after scaling and shifting the spectrum, is given for comparison (green dashed line). (b) Absorption spectrum of the (23,7) SWNT in the $1.45-1.8 \mathrm{eV}$ range; the symbols are the same as in (a).

In the band-to-band model, the imaginary part of the susceptibility is [14]

$$
\chi^{\prime \prime}(E)=\left(\frac{E_{p}}{E}\right)^{2} \frac{\sqrt{\eta+\sqrt{1+\eta^{2}}}}{\sqrt{1+\eta^{2}}},
$$

where $\eta=\left(E-E_{33}\right) / \Gamma_{33}$ and $E_{p}=\hbar \omega_{p}\left(\omega_{p}\right.$ is the plasma frequency).

As in Ref. [14], the real part of the susceptibility is then obtained using the Kramers-Kronig transform. The quality of the fit of the experimental data is better with the excitonic model $\left[R^{2}=0.97\right.$; Fig. 3(a), blue solid line] than with the simplified band-to-band model $\left[R^{2}=0.94\right.$; Fig. 3(a), red dotted line]. As shown in previous studies [14], this could be sufficient to identify the nature of the $E_{33}$ as excitonic. Indeed, contrary to REP, the full Rayleigh spectrum is recorded in one single experiment which lowers the experimental error. 
The values derived in the excitonic model, $E_{33}=1.659 \pm$ $0.005 \mathrm{eV}$ and $\Gamma_{33}=30 \pm 2 \mathrm{meV}$, are in agreement with those found with the fit of the REPs. It should be noted that the fit of the $E_{33}$ Rayleigh peak within the band-to-band model provides a value of the damping parameter $\Gamma_{33}$ close to $15 \pm$ $2 \mathrm{meV}$, which is two times smaller than the one found using the excitonic model [see Fig. 3(a), dotted line]. This value of $\Gamma_{33}$ agrees with the one derived from the fit of the REPs using the same band-to-band approach (Table I).

The Rayleigh spectrum obtained with the full calculation in the band-to-band model is shown for comparison in Fig. 3(a) (green dashed line). In this case, $\Gamma_{33}$ is fixed at $16 \mathrm{meV}$, and the intensity is scaled and rigidly shifted in energy to match the experimental $E_{33}$ transition. Under these assumptions, the simplified and the full band-to-band models give close Rayleigh spectra.

The $E_{33}$ region of the absorption spectrum of the $(23,7)$ SWNT is displayed in Fig. 3(b) (circles). The experimental absorption data are fitted using the imaginary part of the same susceptibilities as for Rayleigh scattering, namely, Eq. (3) for the excitonic model and Eq. (4) for the band-to-band model. The Gaussian spectral profile of the laser used in the absorption experiments, with a half width at half maximum of $7 \mathrm{meV}$, has been taken into account through convolution of $\operatorname{Im} \chi(E)$ by a Gaussian.

The $E_{33}$ absorption peak is excellently reproduced by the excitonic model $\left(R^{2}=0.99\right)$ [12]. The fitted transition energy is $E_{33}=1.664 \pm 0.005 \mathrm{eV}$, and the value of the damping parameter is $\Gamma_{33}=26 \pm 2 \mathrm{meV}$. However, the fit of the $E_{33}$ absorption peak in the simplified band-to-band model clearly fails, with $R^{2}=0.77, E_{33}=1.644 \pm 0.010 \mathrm{eV}$, and $\Gamma_{33}=13 \pm 3 \mathrm{meV}$ [Figure 3(b), red dotted line, and Table I]. Figure 3(b) also shows the calculated profile of the $E_{33}$ absorption peak in the full band-to-band model (green dashed line). The latter is obtained, like before, with $\Gamma_{33}$ fixed at $16 \mathrm{meV}$ and scaling and shifting the spectrum. The simplified and the full band-to-band profiles are very close. In addition, for absorption as well as for Rayleigh scattering, we also checked that the fits of both spectra undergo only minute changes when a complete derivation of the band-to-band susceptibility is performed based on Kim et al.'s work in the three-dimensional semiconductor case [39] with Lorentzian broadening (not shown). Investigating whether Gaussian broadening [39] is relevant in the case of nanotubes is beyond the scope of this paper. In conclusion, the absorption data unambiguously support the excitonic nature of the $E_{33}$ transition of the (23,7) SWNT [12].

On the basis of the analysis of all these experimental data obtained for the same individual $(23,7)$ SWNT, we find that the absorption, Rayleigh, and Raman experimental data are best fitted by considering the $E_{33}$ transition as excitonic. The values of $E_{33}$ and $\Gamma_{33}$ derived from the fit of the three sets of data are in very close agreement (Table I).

\section{CONCLUSION}

The impossibility to determine unambiguously the nature of the $E_{33}$ transition of the $(23,7)$ SWNT, namely, excitonic or band to band, based only on the experimental resonance excitation profiles is confirmed [22]. Conversely, with the excitonic nature of the optical transition being well established by absorption or Rayleigh spectroscopy, we demonstrate that the fit of the REPs using the excitonic model provides a good evaluation of the transition energy $E_{i i}$ and damping parameter $\Gamma_{i i}$ of the optical transitions in SWNTs. In summary, combining absorption, Rayleigh, and Raman spectroscopy on the same individual index-identified SWNT appears to be an efficient method to determine unambiguously the intrinsic features of its optical transitions. The same approach could be used to determine the nature and the features of the optical transitions of individual index-identified double-wall carbon nanotubes.

\section{ACKNOWLEDGMENTS}

H.N.T., M.P., A.A.Z., J.-L.S., and J.-R.H. acknowledge financial support from the ANR GAMBIT project, Grant No. ANR-13-BS10-0014 of the French Agence Nationale de la Recherche, and the International project of Scientific Cooperation with Vietnam (CNRS PICS 6457). We acknowledge the Dr. T. X. Tinh for the synthesis of individual nanotubes. J.-C.B., A.A., A.S.-M., F.V and N.D.F. acknowledge financial support from ANR programme P2N under Contract No. ANR11-NANO-025. V.N.P. acknowledges financial support from Project 316309: INERA - "Research and Innovation Capacity Strengthening of ISSP-BAS in Multifunctional Nanostructures." The TEM studies were conducted at the Laboratorio de Microscopias Avanzadas, Instituto de Nanociencia de Aragon, Universidad de Zaragoza, Spain. Some of the research leading to these results has received funding from the European Union Seventh Framework Programme under Grant Agreement No. 312483- ESTEEM2 (Integrated Infrastructure Initiative -I3). R.A. gratefully acknowledges the support from the Spanish Ministerio de Economia y Competitividad (FIS201346159-C3-3-P) and from the European Union H2020 program ETN project "Enabling Excellence" Grant Agreement No. 642742.
[1] M. Dresselhaus, G. Dresselhaus, R. Saito, and A. Jorio, Annu. Rev. Phys. Chem. 58, 719 (2007).

[2] T. Ando, J. Phys. Soc. Jpn. 66, 1066 (1996).

[3] C. L. Kane and E. J. Mele, Phys. Rev. Lett. 93, 197402 (2004).

[4] V. Perebeinos, J. Tersoff, and P. Avouris, Phys. Rev. Lett. 94, 027402 (2005).
[5] R. B. Capaz, C. D. Spataru, S. Ismail-Beigi, and S. G. Louie, Phys. Rev. B 74, 121401 (2006).

[6] K. Sato, R. Saito, J. Jiang, G. Dresselhaus, and M. S. Dresselhaus, Phys. Rev. B 76, 195446 (2007).

[7] F. Wang, G. Dukovic, L. E. Brus, and T. F. Heinz, Science 308, 838 (2005). 
[8] J. Maultzsch, R. Pomraenke, S. Reich, E. Chang, D. Prezzi, A. Ruini, E. Molinari, M. S. Strano, C. Thomsen, and C. Lienau, Phys. Rev. B 72, 241402 (2005).

[9] J. Lefebvre and P. Finnie, Phys. Rev. Lett. 98, 167406 (2007).

[10] S. Berciaud, L. Cognet, P. Poulin, R. B. Weisman, and B. Lounis, Nano Lett. 7, 1203 (2007).

[11] F. Wang, D. J. Cho, B. Kessler, J. Deslippe, P. J. Schuck, S. G. Louie, A. Zettl, T. F. Heinz, and Y. R. Shen, Phys. Rev. Lett. 99, 227401 (2007).

[12] J.-C. Blancon, M. Paillet, H. N. Tran, X. T. Than, S. A. Guebrou, A. Ayari, A. San Miguel, N.-M. Phan, A. A. Zahab, J.-L. Sauvajol, N. Del Fatti, and F. Vallée, Nat. Commun. 4, 2542 (2013).

[13] M. Y. Sfeir, F. Wang, L. Huang, C.-C. Chuang, J. Hone, S. P. O'Brien, T. Heinz, and L. E. Brus, Science 306, 1540 (2004).

[14] S. Berciaud, C. Voisin, H. Yan, B. Chandra, R. Caldwell, Y. Shan, L. E. Brus, J. Hone, and T. F. Heinz, Phys. Rev. B 81, 041414 (2010).

[15] K. Liu, J. Deslippe, F. Xiao, R. B. Capaz, X. Hong, S. Aloni, A. Zettl, W. Wang, X. Bai, S. G. Louie, E. Wang, and F. Wang, Nat. Nanotechnol. 7, 325 (2012).

[16] H. Lin, J. Lagoute, V. Repain, C. Chacon, Y. Girard, J.-S. Lauret, F. Ducastelle, A. Loiseau, and S. Rousset, Nat. Mater. 9, 235 (2010).

[17] L. Luo, I. Chatzakis, A. Patz, and J. Wang, Phys. Rev. Lett. 114, 107402 (2015).

[18] M. Paillet, T. Michel, J. C. Meyer, V. N. Popov, L. Henrard, S. Roth, and J.-L. Sauvajol, Phys. Rev. Lett. 96, 257401 (2006).

[19] A. Débarre, M. Kobylko, A.-M. Bonnot, A. Richard, V. N. Popov, L. Henrard, and M. Kociak, Phys. Rev. Lett. 101, 197403 (2008).

[20] T. Michel, M. Paillet, D. Nakabayashi, M. Picher, V. Jourdain, J. C. Meyer, A. A. Zahab, and J.-L. Sauvajol, Phys. Rev. B 80, 245416 (2009).

[21] K. Liu, W. Wang, M. Wu, F. Xiao, X. Hong, S. Aloni, X. Bai, E. Wang, and F. Wang, Phys. Rev. B 83, 113404 (2011).

[22] C. Thomsen and S. Reich, Top. Appl. Phys. 108, 115 (2007), and references therein.
[23] T. X. Tinh, V. C. Nguyen, V. Jourdain, M. Paillet, D.-Y. Kim, J.-L. Sauvajol, T. T. T. Ngo, and N. M. Phan, J. Exp. Nanosci. 6, 547 (2011).

[24] R. Arenal, P. Loethman, M. Picher, X. T. Than, M. Paillet, and V. Jourdain, J. Phys. Chem. C 116, 14103 (2012).

[25] D. Christofilos, J.-C. Blancon, J. Arvanitidis, A. San Miguel, A. Ayari, N. Del Fatti, and F. Vallée, J. Phys. Chem. Lett. 3, 1176 (2012).

[26] M. Paillet, F. Meunier, M. Verhaegen, S. Blais-Ouellette, and R. Martel, Rev. Sci. Instrum. 81, 053111 (2010).

[27] J. Lefebvre and P. Finnie, Nano Res. 4, 788 (2011).

[28] G. Bussi, J. Menéndez, J. Ren, M. Canonico, and E. Molinari, Phys. Rev. B 71, 041404 (2005).

[29] J. G. Duque, H. Telg, H. Chen, A. K. Swan, A. P. Shreve, X. Tu, M. Zheng, and S. K. Doorn, Phys. Rev. Lett. 108, 117404 (2012).

[30] A. Jorio, A. G. Souza Filho, G. Dresselhaus, M. S. Dresselhaus, R. Saito, J. H. Hafner, C. M. Lieber, F. M. Matinaga, M. S. S. Dantas, and M. A. Pimenta, Phys. Rev. B 63, 245416 (2001).

[31] C. Fantini, A. Jorio, M. Souza, M. S. Strano, M. S. Dresselhaus, and M. A. Pimenta, Phys. Rev. Lett. 93, 147406 (2004).

[32] H. Telg, J. Maultzsch, S. Reich, F. Hennrich, and C. Thomsen, Phys. Rev. Lett. 93, 177401 (2004).

[33] J. G. Duque, H. Chen, A. K. Swan, A. P. Shreve, S. Kilina, S. Tretiak, X. Tu, M. Zheng, and S. K. Doorn, ACS Nano 5, 5233 (2011).

[34] L. G. Moura, M. V. O Moutinho, P. Venezuela, C. Fantini, A. Righi, M. S. Strano, and M. A. Pimenta, Phys. Rev. B 89, 035402 (2014).

[35] M. Canonico, G. B. Adams, C. Poweleit, J. Menéndez, J. B. Page, G. Harris, H. P. van der Meulen, J. M. Calleja, and J. Rubio, Phys. Rev. B 65, 201402 (2002).

[36] Y. Yin, A. N. Vamivakas, A. G. Walsh, S. B. Cronin, M. S. Ünlü, B. B Goldberg, and A. K. Swan, Phys. Rev. Lett. 98, 037404 (2007).

[37] Y. Yin, A. G. Walsh, A. N. Vamivakas, S. B. Cronin, D. E. Prober, and B. B Goldberg, Phys. Rev. B 84, 075428 (2011).

[38] V. N. Popov and P. Lambin, Phys. Rev. B 73, 165425 (2006).

[39] C. C. Kim, J. W. Garland, H. Abad, and P. M. Raccah, Phys. Rev. B 45, 11749 (1992). 